\title{
Energy doubler for a linear collider
}

\author{
S. Lee, T. Katsouleas, and P. Muggli \\ University of Southern California, Los Angeles, California 90089
}

\author{
W. B. Mori, C. Joshi, R. Hemker, E. S. Dodd, C. E. Clayton, K. A. Marsh, B. Blue, and S. Wang \\ University of California, Los Angeles, Los Angeles, California 90095 \\ R. Assmann, F. J. Decker, M. Hogan, R. Iverson, and D. Walz \\ Stanford Linear Accelerator Center, Stanford University, Stanford, California 94309
}

(Received 9 August 2001; published 17 January 2002)

\begin{abstract}
The concept of using short plasma sections several meters in length to double the energy of a linear collider just before the collision point is proposed and modeled. In this scenario the beams from each side of a linear collider are split into pairs of microbunches with the first driving a plasma wake that accelerates the second. The luminosity of the doubled collider is maintained by employing plasma lenses to reduce the spot size before collision.
\end{abstract}

DOI: 10.1103/PhysRevSTAB.5.011001

PACS numbers: 41.75.Lx, 52.75.-d

At 2 miles long and $50 \mathrm{GeV}$, the Stanford Linear Collider (SLC) is the highest energy linear accelerator in the world, and the only linear collider. Along with the Large Electron Positron Collider (LEP) at CERN, it has succeeded in unveiling much of the detailed physics of the standard model of elementary particles and fields. However, the Higgs boson and the ultimate test of the standard model appear now to lie above $100 \mathrm{GeV}$ and therefore out of the reach of the SLC. The results of the last runs of LEP were suggestive of the fact that the discovery of the Higgs may have been just beyond the reach of that machine [1,2]. In this report, we describe a scenario for doubling the energy of a collider by using a plasma wake field accelerator section several meters long placed at the end of each beam line just before the collision point. Such a doubling scheme could be used to extend the high-energy physics reach of the SLC or a future linear collider.

The concept of a plasma wake field accelerator has received considerable attention recently [3-7]. In a plasma wake field accelerator, the space charge of a particle bunch displaces the electrons of a preformed quiescent plasma to produce a large plasma wake field that can accelerate a subsequent bunch at a very high rate. In this report, 3D simulation models are used to show that the amplitude of the accelerating wake scales with the inverse square of the bunch length and that this scaling continues to hold for pa- rameters far exceeding the linear theory from which it is derived. This leads us to propose the concept of a plasma afterburner - a specifically designed plasma that accelerates as well as focuses each beam from a linear collider in a single, short, final stage. Finally, we outline the critical issues that remain to be addressed in order to realize this concept.

The afterburner concept is illustrated schematically in Fig. 1. Electrons and positrons are accelerated to the collider's nominal operating energy (e.g., $50 \mathrm{GeV}$ for the SLC example), overcompressed to form two microbunches each, then the trailing half-bunches are doubled in energy over a few meters in the plasma afterburner. To sustain the luminosity at the interaction point (IP) at the nominal level of the original collider without the plasma, the reduction in number of particles must be offset by a smaller spot size at the IP. Reduction of the spot size is possible in the strong focusing fields of the plasma; thus, higher density plasma lenses are added to the design just before the interaction point.

To guide the discussion of the simulations to follow, we begin by reviewing key features of plasma wake field excitation in linear theory [8]. The linear response of a plasma to a Gaussian bunch is optimized when the plasma density $\left(n_{o}\right)$ is chosen such that the bunch length and plasma wavelength are matched; more precisely, for $k_{p} \sigma_{z} \cong \sqrt{2}$,

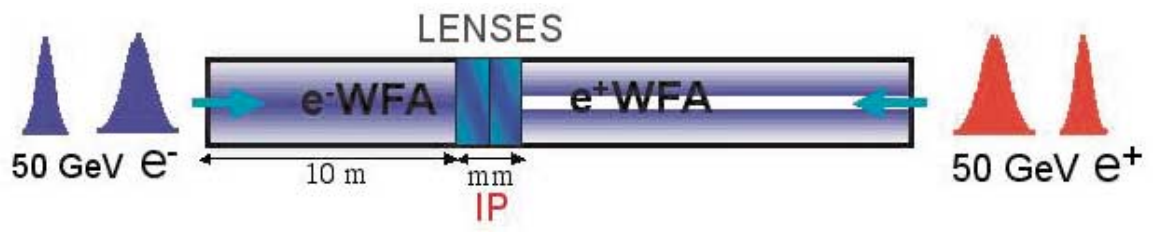

FIG. 1. (Color) Schematic of the plasma afterburner concept. Plasma wake field acceleration (WFA) sections are placed at the interaction point (IP); short plasma lens sections of higher density further focus the beams before collision. Note that the WFAs are not symmetric; the positron section may be longer to reach the same energy and may have a channel to enhance the wake. 


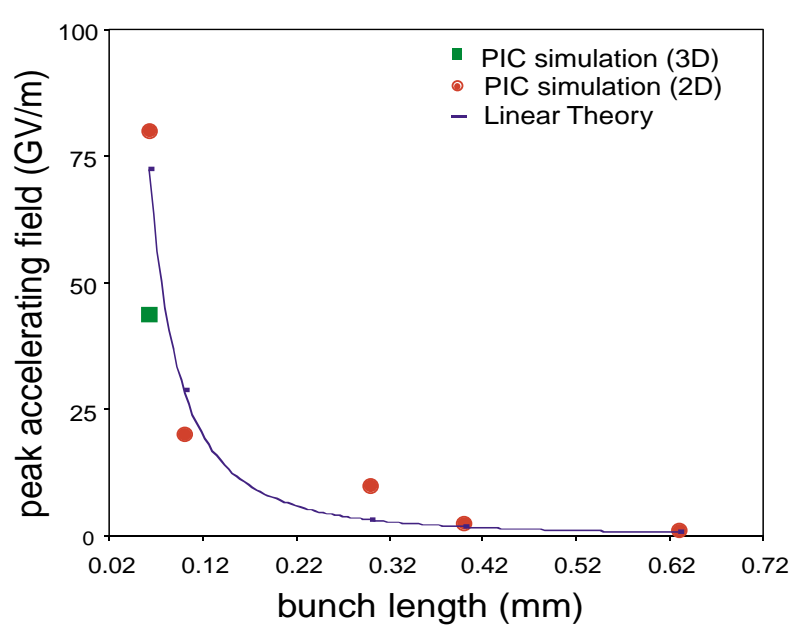

FIG. 2. (Color) Particle-in-cell simulation results for the peak plasma wake amplitude versus bunch length. The solid curve shows the scaling from linear theory [Eq. (1)]. In these simulations, the cell size was kept at $0.1 c / \omega_{p}$ in the transverse direction, $0.05 c / \omega_{p}$ longitudinally, nine particles/cell (4 in $\left.3 \mathrm{D}\right)$, and the plasma density was adjusted to maintain $k_{p} \sigma_{z} \cong \sqrt{2}$. The width of the system was adjusted to ensure that the plasma blowout did not reach the walls (typically $6 c / \omega_{p}$ ).

where $\sigma_{z}$ is the Gaussian bunch length and $k_{p}=\omega_{p} / c$ is 1 over the plasma skin depth $\left(c / \sqrt{4 \pi n_{o} e^{2} / m}\right)$. In this case, the wake amplitude scales linearly in the bunch charge and inversely with the square of the bunch length:

$$
e E_{o} \approx 240(\mathrm{MeV} / \mathrm{m})\left(\frac{N}{4 \times 10^{10}}\right)\left(\frac{0.6 \mathrm{~mm}}{\sigma_{z}}\right)^{2}
$$

This strong bunch length dependence is a primary motivator for this work. It suggests that simply decreasing the bunch length by a factor of 10 from present SLAC parameters could increase the wake field amplitude by 100 . However, this is strictly valid only within the limits of linear theory, i.e., $n_{b} \ll n_{o}$. For the parameters typical of the SLC bunches and the plasmas of interest for high gradient acceleration, this inequality is not fulfilled. Thus, we turn instead to numerical simulations.

To study the wake field excitation scaling laws in the nonlinear regime of $n_{b}>n_{o}$, we employ the 3D fully self-consistent and object-oriented model OSIRIS. The simulation model has been described in more detail elsewhere $[9,10]$. It follows $10^{6}-10^{8}$ particles on a $2 \mathrm{D}$ or 3D moving mesh of $10^{5}-10^{6}$ cells. The beam and plasma particles move according to the Lorentz force and their self-consistent electromagnetic fields, found by solving Maxwell's equations on the grid. This code and others have been used previously to model nonlinear wake fields excited by electron bunches with densities a few times the plasma density [1]. Here we extend that work by considering much shorter bunches for which the beam density far exceeds the plasma density.

Figure 2 shows the results of several simulation runs to measure peak wake amplitude versus bunch length. For each case, the number of particles is kept fixed $(N=$ $4 \times 10^{10}$ ) and the plasma density is adjusted to match to the bunch length. The solid curve is the linear theory prediction. Although the wakes are highly nonlinear, the linear theory expression for the amplitude is in good agreement with the simulations up to values of $E / E_{D} \sim 5$, where $E_{D}$ is the Dawson nonrelativistic wave breaking field $\left(\sim \sqrt{n_{o}} \mathrm{~V} / \mathrm{cm}\right)[11]$. We comment that the optimal plasma density for a given bunch length is slightly higher than the linear theory expression above. This is because of the nonlinear frequency shift of the plasma wave at large amplitude. This shift toward lower frequency arises from the relativistic mass increase of the oscillating plasma electrons.

A sample snapshot of the plasma wake fields excited by a short electron bunch is shown in Fig. 3 a. In this example, the electron bunch consisted of a $3 \times 10^{10}$ electron in a bi-Gaussian distribution of radius $\sigma_{r}=25 \mu \mathrm{m}$ and length $\sigma_{z}=63 \mu \mathrm{m}$ (10 times shorter than is typical of the current SLC beam). The plasma density was chosen to be $1.8 \times 10^{16} \mathrm{~cm}^{-3}$ corresponding approximately to the plasma matching discussed above. The peak beam density

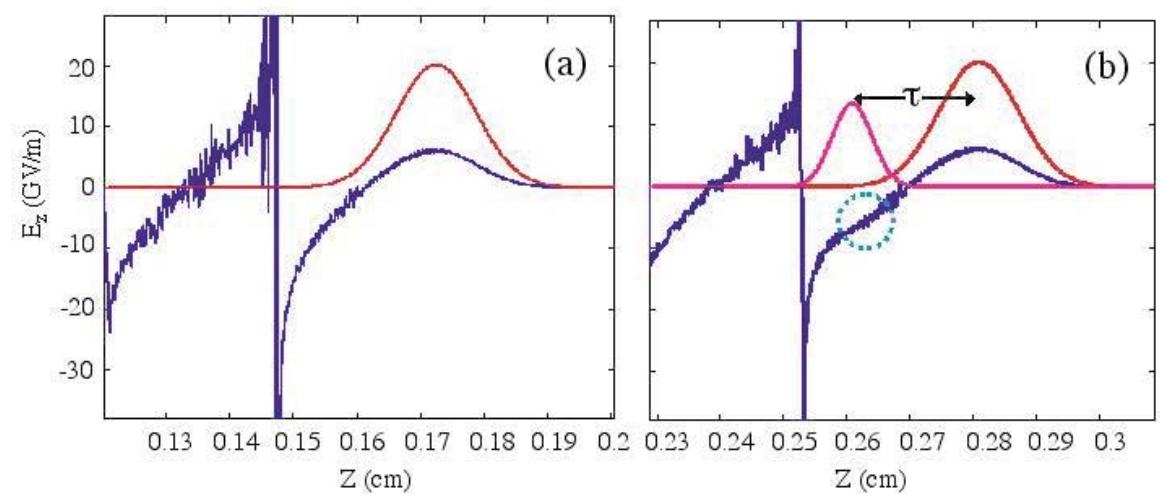

FIG. 3. (Color) Longitudinal plasma wake fields on the axis for the beam profiles shown. (a) Drive bunch only and (b) drive bunch and trailing bunch separated by delay $\tau$. Note flattening of wake due to beam loading shown in the dotted area. 
is $4.8 \times 10^{16} \mathrm{~cm}^{-3}$, well above the plasma density. Accordingly, the plasma response is very nonlinear as seen in Fig. 3. A large spike in the longitudinal field is apparent where the plasma electrons that were expelled by the beam have returned to the axis. The peak accelerating field (i.e., amplitude of the spike) is $80 \mathrm{GeV} / \mathrm{m}$ for this case. The focusing force is nearly constant ahead of the spike at the value $2 \pi n_{o} e^{2} r$, as one would obtain from the bare space charge of a positive ion column.

The results in Figs. 2 and 3 are suggestive. With fields of several tens of $\mathrm{GeV} / \mathrm{m}$, it would appear to be possible to double the energy of a $50 \mathrm{GeV}$ collider with plasma afterburner sections that are only a few meters long. The realization of this promise is contingent upon a number of physics and technology issues. These are described next. They include (i) beam loading (generating and phasing very short bunches), (ii) transverse beam dynamics (betatron oscillations and emittance growth as well as electron hose instability), (iii) plasma source development, (iv) positron acceleration, and (v) modeling.

Beam loading and phasing. - We consider a two bunch system consisting of a driver bunch and a trailer bunch separated by a small distance such that the trailer sits near the peak wake field of the driver. One possible means to achieve a double-bunch arrangement in the SLC is to use a bunch compression system operating in a mode so as to overbunch a single bunch. To obtain a modest energy spread of the accelerated bunch requires that the second bunch not only be properly phased but also shorter than the first. To be specific, we take the following parameters for a round beam example. Drive beam number and size: $N=3 \times 10^{10}, \sigma_{z}=63 \mu \mathrm{m}, \sigma_{r}=25 \mu \mathrm{m}$; trailer beam: $\quad N=1 \times 10^{10}, \quad \sigma_{z}=32 \mu \mathrm{m}, \quad \sigma_{r}=25 \mu \mathrm{m}$; plasma density and length: $n_{o}=1.8 \times 10^{16} \mathrm{~cm}^{-3}$, $L \sim 7 \mathrm{~m}$.

The spacing between the microbunches is determined empirically from simulations. Figure $3 b$ shows a snapshot of the wake due to the two bunches. By comparing to the unloaded wake in Fig. 3a, we see the absorption of the wake due to the nonlinear beam loading by the second bunch. We performed several simulation runs with different phasing of the load. Increasing the phase delay increases the peak energy and the energy spread. The delay for this case was 0.6 ps giving an average energy gain rate of $8 \mathrm{GeV} / \mathrm{m}$ with a FWHM spread of $20 \%$. We note that the maximum decelerating field on the driving bunch is $7 \mathrm{GeV} / \mathrm{m}$. Thus neglecting deformation of the bunches, they could propagate a distance of $7 \mathrm{~m}$, imparting an energy of $56 \mathrm{GeV}$ to the trailing bunch.

We note that this beam loading example is not optimized. For example, slightly more charge in the bunch load could further flatten the wake at the beam load (see Fig. 3b) and reduce the energy spread. Moreover, it is possible to more than double the energy of the trailing bunch by increasing the phase delay of the bunch load so as to sample the larger electric field near the spike. However, the steepening of the wave form near the spike requires the beam load to be even shorter and narrower. Naturally, fewer particles can be supported by the field energy there (as is also apparent from energy conservation), so one must choose how much to trade off luminosity for higher energy.

Transverse beam dynamics. - A great deal of information about transverse beam propagation issues has been learned from a recent meter-long plasma wake field experiment at SLAC known as E-157 [3]. Of primary concern are betatron oscillations and hose instabilities. While there were nominally three to five betatron oscillations in E-157, there will be roughly 100 over the length of an afterburner. If there is phase mixing due to longitudinal and transverse focusing aberrations, there will be an unrecoverable emittance growth. To prevent this, the beta functions of the beam and the plasma focusing must be matched [4]. The matching condition for electrons is

$$
\gamma \varepsilon^{2} / \sigma^{4}=2 \pi n_{o} r_{e},
$$

where $\varepsilon$ is the beam emittance, $\gamma$ is its energy, $\sigma$ its spot size, and $r_{e}$ is the classical electron radius. This will require beams to be of the order of $1 \mu \mathrm{m}$ in radius at the plasma entrance $(1.3 \mu \mathrm{m}$ for a normalized emittance of $10 \mathrm{~mm}$ mrad at $50 \mathrm{GeV}$ ) or an adiabatic plasma transition section. The modeling of submicron beams with particle-in-cell simulations requires a resolution that challenges the capability of current computers.

A second transverse issue is the electron hose instability. Simple analytic estimates $[12,13]$ suggest that there may be of the order of $10 e$-foldings of hose growth for afterburner parameters. However, the electron hose instability was not observed experimentally in E-157 even though up to three $e$-foldings were predicted. The experiment was in agreement with simulations that showed that E- 157 is near the margin for hose instability. Further work is needed to study hosing for afterburner parameters and to address stabilization mechanisms such as transverse plasma gradients.

A third transverse issue arises from the plasma lenses at the end of the acceleration sections. It is straightforward to estimate from the envelope equation for an electron beam [14] the spot size reduction possible by a plasma lens [15]. For a plasma lens of density $n_{l}$ satisfying $n_{b} \gg n_{l} \gg n_{o}$, and assuming that the normalized emittance is preserved in the plasma, the spot size can be compressed by a factor of $\sqrt{n_{l} / n_{o}}$. For the example above, a short (approximately $2 \mathrm{~mm}$ ) plasma section of density $2 \times 10^{18} \mathrm{~cm}^{-3}$ would reduce the spot size from the order of $1 \mu \mathrm{m}$ to the order of $100 \mathrm{~nm}$.

Plasma source development. - The plasma parameters in the afterburner example above somewhat exceed the state of the art in terms of the production of long highdensity plasmas. Just how the plasma density can be stepped up for the plasma lenses and how the walls of the plasma device can be made compatible with the detector 
design are also unresolved issues. An important research direction then is the development of new plasma production techniques. Beam ionization of gas and resonant laser ionization are promising possibilities requiring experimental investigation [16].

Positron acceleration. - In the nonlinear regime here, the wake excitation by positron beams is not symmetric to the electron case. In recent work [17], simulations showed that positron wakes are typically smaller than electron wakes due to phase mixing of the arrival time of plasma electrons sucked into the beam axis. However, the positron wakes can become comparable to the electron wakes if a hollow or ring-shaped plasma of radius $c / \omega_{p}$ is used. Just such a profile was demonstrated by blocking the center of the ionizing laser at the E-157 laboratory. Experimental investigation of nonlinear positron wakes and positron propagation and acceleration is of great importance for further assessment of this scheme.

The work presented here suggests a promising path for achieving a breakthrough in advanced accelerator development. Preliminary simulations support the possibility of an energy doubler for linear colliders based on short plasma wake field sections placed just before the collision point. A concerted research effort to demonstrate control of transverse instabilities, develop plasma sources of sufficient length and density, and produce short microbunches is the key to realizing this concept.

\section{ACKNOWLEDGMENTS}

This work was supported by U.S. Department of Energy Contracts No. DE-FG03-92ER40745, No. DE-FG0398DP00211, No. DE-FG03-92ER40727, and No. DEAC03-765F00515, NSF Contracts No. ECS-9632735 and
No. DMS-9722121, and LLNL Contract No. W07405ENG48. We are indebted to the late John Dawson for his early vision of boosting the energy of linear colliders with plasma wake field sections, and we acknowledge stimulating conversations with David Sutter at the 1999 Particle Accelerator Conference and Marty Breidenbach at SLAC that prompted us to undertake this design study. Ongoing discussions with Robert Siemann are gratefully acknowledged.

[1] O. Morton, Science 289, 2014 (2000).

[2] O. Morton, Science 290, 1274 (2000).

[3] M. J. Hogan et al., Phys. Plasmas 7, 224 (2000).

[4] P. Muggli et al., Nature (London) 411, 43 (2001).

[5] S. Lee, T. Katsouleas, R. Hemker, and W. B. Mori, Phys. Rev. E 61, 7014 (2000).

[6] R. G. Hemker, W. B. Mori, S. Lee, and T. Katsouleas, Phys. Rev. ST Accel. Beams 3, 061301 (2000).

[7] N. Barov, J. B. Rosenzweig, M. E. Conde, W. Gai, and J. G. Power, Phys. Rev. ST Accel. Beams 3, 011301 (2000).

[8] R. Keinigs and M. E. Jones, Phys. Fluids 30, 252 (1987).

[9] R. G. Hemker, F. S. Tsung, V.K. Decyk, W. B. Mori, S. Lee, and T. Katsouleas, in Proceedings of the 1999 Particle Accelerator Conference, New York (IEEE, Piscataway, NJ, 1999), p. 3672.

[10] R. Hemker, Ph.D thesis, University of California, Los Angeles, 2000.

[11] J. M. Dawson, Phys. Rev. 133, 383 (1959).

[12] A. Geraci and D. Whittum, Phys. Plasmas 7, 3431 (2000).

[13] E. Dodd et al., Phys. Rev. Lett. (to be published).

[14] C. Clayton et al., Phys. Rev. Lett. (to be published).

[15] P. Chen, Part. Accel. 20, 171 (1985).

[16] C. Joshi (private communication).

[17] S. Lee et al., Phys. Rev. E 64, 045501 (2001). 